\title{
VLBA Observations of Hi in the Archetype Compact Symmetric Object B2352+495
}

\author{
E. D. Araya ${ }^{1,2,3}$, C. Rodríguez ${ }^{2}$, Y. Pihlström ${ }^{2,4}$, G. B. Taylor ${ }^{2,4}$, S. Tremblay ${ }^{2}$ \\ and \\ R. C. Vermeulen ${ }^{5}$
}

\begin{abstract}
B2352+495 is a prototypical example of a Compact Symmetric Object (CSO). It has a double radio lobe symmetrically located with respect to a central flat spectrum radio core (the location of the AGN) and has a physical extent of less than 200 pc. In this work we report VLBA observation of $21 \mathrm{~cm} \mathrm{H} \mathrm{I} \mathrm{absorp-}$ tion toward B2352+495 to investigate the properties of this remarkable radio source, in particular, to explore whether the radio emission can be confined by circumnuclear material (frustration scenario) or whether the source is likely to be young. We confirmed the two H I absorption features previously detected toward B2352+495 - a broad line nearly centered at the systemic velocity of the galaxy and a narrow redshifted component. The atomic gas from the broad absorption component is likely associated with circumnuclear material, consistent with the current paradigm of clumpy HI distribution in toroidal structures around supermassive black holes.
\end{abstract}

Subject headings: galaxies: active - galaxies: individual (B2352+495) - radio lines: galaxies

\footnotetext{
${ }^{1}$ Jansky Fellow of the National Radio Astronomy Observatory, P.O. Box 0, Socorro, NM 87801.

${ }^{2}$ Department of Physics and Astronomy, MSC07 4220, University of New Mexico, Albuquerque, NM 87131.

${ }^{3}$ Physics Department, Western Illinois University, 1 University Circle, Macomb, IL 61455.

${ }^{4}$ Adjunct Astronomer, National Radio Astronomy Observatory.

${ }^{5}$ ASTRON, Netherlands Foundation for Research in Astronomy, PO Box 2, 7990 AA Dwingeloo, The Netherlands.
} 


\section{Introduction}

Compact Symmetric Objects (CSOs) are characterized by compact bright radio emission at scales $<1 \mathrm{kpc}$ with lobes on both sides of a central engine (Wilkinson et al. 1994). CSOs tend to be associated with bright elliptical galaxies, although some appear to be associated with quasars and even spiral galaxies (Readhead et al.1996; Augusto et al. 2006; Perlman et al. 1996). Many CSOs have an overall GigaHertz-Peaked Spectrum (GPS), and the cores are often undetected at frequencies of $5 \mathrm{GHz}$ or higher (Taylor et al. 1996). CSOs are very luminous radio sources, significantly brighter (by more than a factor of 10) than the luminosity threshold between FR I and II radio galaxies (i.e., $\sim 2 \times 10^{25} \mathrm{~W} \mathrm{~Hz}^{-1} \mathrm{sr}^{-1}$ at $178 \mathrm{MHz}$, Fanaroff \& Riley 1974; Readhead et al. 1996).

It has been proposed that the small source size could be caused by "frustration" of the radio jets, which fail to escape from a dense galactic medium (e.g., van Breugel et al. 1984; O'Dea et al. 1991; De Young 1993). However, a different scenario is currently preferred: CSOs may be young progenitors $\left(\lesssim 10^{3}-10^{5}\right.$ years) of powerful extended radio sources (e.g., Phillips \& Mutel 1982; Augusto et al. 2006; Augusto 2007). Based on XMM-Newton observations of five CSOs, Vink et al. (2006) found that their absorption column density $\left(N_{H} \sim 10^{22} \mathrm{~cm}^{-2}\right)$ is on average greater than that of broad-line radio galaxies, but similar to column densities observed toward narrow-line and other types of radio galaxies (NLRGs, RG; see Sambruna et al. 1999 for definitions). These results argue against the frustration scenario because much higher X-ray absorption column densities than found by Vink et al. (2006) are expected if the expansion of the radio jets were confined (see also Guainazzi et al. 2006). In contrast, the dynamical age of CSOs measured from the expansion velocity of hotspots in the radio lobes supports the youth hypothesis (Taylor et al.2000, Polatidis \& Conway 2003). The youth interpretation is also supported by limits on molecular gas content (O'Dea et al. 2005), H I observations (Pihlström et al.2003), and under-luminous O III line emission (Vink et al. 2006).

The study of CSOs is therefore fundamental for the understanding of the formation and evolution of radio galaxies. In addition, CSOs are good candidates to investigate the fueling of supermassive black holes given that the compact radio emission enables very high angular resolution observations of circumnuclear gas via absorption studies. In this work we report high sensitivity and high angular resolution observations of $\mathrm{HI}$ toward B2352+495; an archetype of the CSO class. 


\subsection{HI Absorption in Compact Symmetric Objects}

According to Curran et al. (2008), H I absorption associated with sources at redshifts greater than $\sim 0.1$ has been detected toward 37 sources, 13 of them classified as CSOs, GPS, or HFP (High Frequency Peaked) sources. An overview of H I absorption in compact radio sources was published by Vermeulen et al. (2003a). They report an H I detection rate of $33 \%$ based on Westerbork Synthesis Radio Telescope (WSRT) observations of 59 sources (i.e., 19 detections). Of the 59 sources, 11 were classified as CSO by Augusto et al. (2006) and Polatidis \& Conway (2003). From this subsample of 11 CSOs, 6 (54\%) show H I absorption. Other studies show similar results, e.g., Peck \& Taylor (2001) report a CSO H I detection rate of $\sim 50 \%$, and Gupta et al. (2006) report a detection rate of $\sim 45 \%$ in GPS sources (see also Vermeulen 2002).

The optical depth range of the 6 CSOs with H I absorption from Vermeulen et al. (2003a) is 0.2 to $1.7 \%$, with linewidths between 13 and $297 \mathrm{~km} \mathrm{~s}^{-1}$ and column densities between $2.8 \times 10^{19}$ and $2.6 \times 10^{20} \mathrm{~cm}^{-2}$. To estimate H I column densities, Vermeulen et al. (2003a) assumed that the atomic gas was uniformly covering the radio continuum (covering factor of 1) and a spin temperature of $100 \mathrm{~K}$, that may underestimate the spin temperature by more than an order of magnitude if the gas were located at parsec-scales from the AGN. Thus, the column densities reported by Vermeulen et al. (2003a) are lower limits.

Including the results reported in this work, H I imaging at high angular resolution has been reported toward five CSOs (Table 1). We also list in Table 1 all non-CSO AGN and starbursts that have been imaged at high angular resolution in HI, i.e., in interferometric observations using baselines greater than $100 \mathrm{~km}$. In the rest of this section we review the most salient characteristics of the CSOs that have been studied in H I at high angular resolution:

- B1946+708: High angular resolution H I observations were conducted by Peck et al. (1999; see also Peck \& Taylor 2001). They found extended and multi-peaked absorption throughout the radio continuum source. The peak optical depths range between 3 and $7 \%$. The maximum column density $\left(N_{H I} \sim 3 \times 10^{23} \mathrm{~cm}^{-2}\right.$; Peck \& Taylor 2001) was found toward the AGN core, which also shows the greatest velocity dispersion $\left(F W H M \sim 350 \mathrm{~km} \mathrm{~s}^{-1}\right)$. The extent of the H I absorption in B1946+708 implies that the atomic gas is tracing a thick torus (thickness $>80 \mathrm{pc}$ ).

- 4C 31.04: The host galaxy is a bright elliptical at $\mathrm{z}=0.0592$. It was classified as CSO based on VLBI continuum observations that revealed a double radio lobe with a flat spectrum radio core (Cotton et al. 1995; Giovannini et al. 2001, Giroletti et al. 2003; see also Augusto et al.1998). Detection of H I in 4C 31.04 was reported by Mirabel (1990) 
based on Arecibo observations. Mirabel (1990) found two absorption features, a broad $\left(\mathrm{FWHM}=133 \mathrm{~km} \mathrm{~s}^{-1}\right)$ component and a double peaked narrow component $(\mathrm{FWHM}=6$ and $16 \mathrm{~km} \mathrm{~s}^{-1}$ ) redshifted approximately $400 \mathrm{~km} \mathrm{~s}^{-1}$ from the optical velocity. Subsequent VLBI observations by Conway (1999) showed that the broad line covers one of the radio lobes and just partially the second, indicating a sharp edge of the atomic gas torus. The narrow component is detected toward both radio lobes. The maximum optical depth of the lines is $\sim 5$ to $7 \%$. Mirabel (1990) interpreted the narrow absorption as being caused by clouds similar to high velocity H I clouds in our Galaxy.

- 4C 37.11: In contrast to the two previous CSOs, H I in 4C 37.11 has been detected only toward one of the radio lobes. The absorption is characterized by two broad (FWHM $>100 \mathrm{~km}$ $\mathrm{s}^{-1}$ ) H I features from two separated (in line-of-sight and velocity) clouds. The peak optical depth of the HI lines is $\sim 2 \%$. Rodríguez et al. (2009) concluded that the H I absorption likely originates in a thick circumnuclear torus.

- PKS $1413+135:$ As in 4C 37.11, H I absorption is detected only toward one radio lobe (Perlman et al. 2002). The H I optical depth is $\tau \approx 1$ and the line is quite narrow, between 16 and $18 \mathrm{~km} \mathrm{~s}^{-1}$. Perlman et al. (2002) concluded that the H I absorption is likely from a giant molecular cloud in the outer disk of the host galaxy. Although PKS 1413+135 was classified by Perlman et al. (1996) and Gugliucci et al. (2005) as a CSO, it has several characteristics that differ form a typical CSO. In particular, it was originally classified as a BL Lac object based on its optical spectrum (Bregman et al. 1981), the nucleus dominates by more than $50 \%$ of the total cm flux density, the radio emission is variable, and the host appears to be a spiral galaxy (see Perlman et al. 1996).

Finally, two additional sources (B1934-638 and 4C 12.50) are worth discussing in detail. B1934-638 is a compact-double GPS source that has been classified as a CSO (Augusto et al. 2006), however, no flat-spectrum radio core has been detected (Tzioumis et al. 2002). Even though no H I imaging has been reported at high angular resolution, the H I absorption is against the compact radio continuum as shown by detection of absorption with three baselines of the LBA (Véron-Cetty et al. 2000). The absorption is narrow (FWHM $=18 \mathrm{~km}$ $\mathrm{s}^{-1}$ ) and redshifted $260 \mathrm{~km} \mathrm{~s}^{-1}$ from the optical systemic velocity, thus, the H I is probably not located in the nuclear region of the galaxy. We include B1934-638 in Table 1 although no VLBI H I imaging is available and the radio core has not been detected.

In the case of $4 \mathrm{C} 12.50$, high angular resolution $\mathrm{H}$ I absorption observations were reported by Morganti et al. (2004). They found H I absorption against a weak counterjet and concluded that the H I likely traces a jet/cloud interaction and not a circumnuclear torus or disk. This source was classified as a CSO by Lister et al. (2003) despite several atypical characteristics. For instance, while the $1.3 \mathrm{GHz}$ continuum image reveals a S-shaped distribution with a total 
extend of $\sim 300 \mathrm{pc}$ (Morganti et al. 2004), the $15 \mathrm{GHz}$ VLBA observations are consistent with a core-jet morphology plus a weak counterjet (i.e., inconsistent with the typical symmetrical morphology of CSOs). 4C 12.50 has no clear hotspots, it is classified as a CSS (Compact Steep Spectrum), the radio jet exhibits superluminal motions, some isolated features have unusually high $(\sim 60 \%)$ linear polarization in comparison to other CSOs, and more importantly, there appears to be continuity between the $\sim 300$ pc compact radio jets and large scale $(>10 \mathrm{kpc})$ radio lobes (Stanghellini et al.2005). Therefore, we do not consider 4C 12.50 a bonafide member of the CSO class (see Table 1).

\subsection{B2352+495: An Archetype Compact Symmetric Object}

B2352+495 has been studied in detail by a number of authors (e.g., Readhead et al. 1996, Taylor et al. 1996); in this section we summarize the characteristics of this object:

Radio Continuum: The most salient property of B2352+495 is its highly symmetric morphology at radio wavelengths. It exhibits an S-shaped morphology with a total extent of $\sim 120 \mathrm{pc}$ (Readhead et al.1996). High angular resolution radio continuum observations from $610 \mathrm{MHz}$ to $15 \mathrm{GHz}$ reveal symmetric hotspots and lobes; remarkably similar to the morphology of FR II objects (but at much smaller scale). The dynamical age of the system is $\sim 1200 \mathrm{yr}$ based on proper motion studies of the hotspots in the radio lobes (Taylor et al. 2000). Taylor et al. (1996) detected the radio core of the system, i.e., the putative location of the central engine. The core is approximately located symmetrically between the radio lobes, and drives a collimated jet that is brighter toward the northern side of the core. The radio jet is detected up to the brightest radio emission in the system (B1 and B2; Taylor et al 1996; see also §3) which is also located between the two radio lobes. The hotspots in the radio lobes show no superluminal motion (Conway et al.1992). Multi-frequency VLA observations give fractional polarization limits of $<1 \%$ for this source (Rudnick \& Jones 1983). The radio spectral energy distribution of B2352+495 peaks at $\sim 1 \mathrm{GHz}$, thus by definition, it is a GPS object (Conway et al.1992). The total flux density of B2352+495 shows only modest $(\lesssim 20 \%)$ variability on time scales of months; no systematic long term variability was found over a period of $\sim 15$ years (Waltman et al. 1991; Conway et al. 1992).

The radio luminosity is $\sim 10^{26} h^{-2} \mathrm{~W} \mathrm{~Hz}^{-1}$ ( $\nu_{o}=5 \mathrm{GHz}$ at the emitted frame), i.e., some 20 times brighter than the luminosity boundary between FR Is and FR IIs (Readhead et al. 1996).

Optical/Infrared Counterpart: B2352+495 was not detected by IRAS to sensitivity limits well below the corresponding luminosity of Arp 220 (at the redshifted frequency), thus B2352+495 is significantly less luminous than ultra-luminous infrared galaxies (Readhead et 
al. 1996). Snellen et al. (2003) conducted imaging and spectroscopic optical observations of B2352+495. They found that the radio source resides in an optical host in the fundamental plane of elliptical galaxies, i.e., the host is a normal elliptical. Snellen et al. (2003) report a redshift of $0.23790 \pm 0.00016$, stellar velocity dispersion of $201 \pm 17 \mathrm{~km} \mathrm{~s}^{-1}$, and an effective radius of $1.6^{\prime \prime} \pm 0.3^{\prime \prime}(5.8 \pm 1.1 \mathrm{kpc}) 11$ Based on the stellar velocity dispersion, the mass of the supermassive black hole at the center of B2352+495 is of the order of $10^{8} \mathrm{M}_{\odot}$. The absolute magnitude of the galaxy is $M_{V}=-19.8$ with an apparent magnitude of $m_{V}=20.1$ (Readhead et al. 1996). The optical luminosity of B2352+495 is $\sim 0.35 L^{*}$, which is smaller than the typical optical luminosity of Classical Doubles (FR IIs; $L \sim L^{*}$; Owen \& White 1991) and $\mathrm{cD}$ galaxies $\left(L \sim 5-10 L^{*}\right.$; Schechter 1976). The absence of Balmer lines and strong blue continuum in the spectrum demonstrates no starburst activity. In contrast, a number of emission lines are detected typical of active elliptical galaxies (Readhead et al. 1996). The AGN contribution to the total optical continuum is $\lesssim 10 \%$ (Snellen et al. 2003).

X-Ray Properties: High sensitivity XMM-Newton observations of B2352+495 were conducted by Vink et al. (2006). They detected an X-ray source with a luminosity of $4.6 \times$ $10^{42} \mathrm{erg} \mathrm{s}^{-1}(2-10 \mathrm{keV}$ band, ignoring absorption). Based on the X-ray detection, they estimated an intrinsic absorption column density of $(0.66 \pm 0.27) \times 10^{22} \mathrm{~cm}^{-2}$, which is similar to the column densities found toward extended radio galaxies.

Prior HI Observations: Absorption of the HI transition in B2352+495 was first detected by Vermeulen et al. (2003a) based on WSRT observations. The H I absorption in B2352+495 consists of a broad line (FWHM $\left.=82 \mathrm{~km} \mathrm{~s}^{-1}\right)$ and a redshifted narrow component (FWHM $\left.=13 \mathrm{~km} \mathrm{~s}^{-1}\right)$. The peak flux density of the two absorption components is similar $(\sim 40 \mathrm{mJy})$. Preliminary results of the data reported in this paper were discussed by Vermeulen (2002).

\section{Observations}

Very long baseline interferometer (VLBI) observations were conducted on December 01 and 02, 1997, with the NRAO Very Long Baseline Array (VLBA 2 ) and the WSRT. The data were correlated with the VLBA correlator at the P.V.D. Science Operations Center in Socorro, New Mexico. The target source (B2352+495) was observed during approximately 8.5 hours each day $(\sim 17$ hours total $)$ in the $21 \mathrm{~cm} \mathrm{H} \mathrm{I} \mathrm{transition}\left(\nu_{0}=1420.406 \mathrm{MHz}\right)$ which

\footnotetext{
${ }^{1}$ At a redshift of $0.23790,10$ mas correspond to $36.3 \mathrm{pc}$ assuming a cosmology of $H_{0}=73.0 \mathrm{~km} \mathrm{~s}^{-1} \mathrm{Mpc}^{-1}$, $\Omega_{\text {matter }}=0.27, \Omega_{\text {vacuum }}=0.73$.

${ }^{2}$ The VLBA is operated by the National Radio Astronomy Observatory (NRAO), a facility of the National Science Foundation operated under cooperative agreement by Associated Universities, Inc.
} 
is redshifted to a sky frequency of $\nu_{\text {sky }}=1147.40 \mathrm{MHz}(\mathrm{z}=0.23790 \pm 0.00016$, Snellen et al. 2003; $\mathrm{V}_{\text {Hel }}=71321 \pm 48 \mathrm{~km} \mathrm{~s}^{-1}$, optical definition). We observed with a bandwidth of $8 \mathrm{MHz}, 1024$ channels, dual circular polarization, and an initial channel width of $\Delta \nu=$ $7.8 \mathrm{kHz}$ smoothed to a spectral resolution of $15.6 \mathrm{kHz}\left(4.1 \mathrm{~km} \mathrm{~s}^{-1}\right)$. Only eight of the ten VLBA antennas were used because of RFI filters that block access at Fort Davis and Kitt Peak. We observed 3C84 for bandpass calibration.

All data reduction was done in the NRAO package AIPS using standard VLBA spectralline calibration procedures. The WSRT data were significantly affected by RFI and we decided not to use them after careful inspection of the results obtained including and excluding WSRT. We used line-free channels of the B2352+495 observations for fringe fitting. A continuum data set was obtained by averaging the line-free channels of the visibility file; the continuum data were used for self-calibration. The absolute astrometry of the data was therefore lost, and we simply assign the radio continuum peak to the phase tracking center $\left(\mathrm{RA}=22^{\mathrm{h}} 55^{\mathrm{m}} 09.4581^{\mathrm{s}}\right.$, Decl. $\left.=+49^{\circ} 50^{\prime} 08.340^{\prime \prime}, \mathrm{J} 2000\right)$.

Given the non-standard frequency of the observations, a reliable flux density calibration using the $\mathrm{T}_{\text {sys }}$ method was not possible, thus we scaled the flux density to a total radio continuum emission of $2.55 \mathrm{Jy}$ at $1.14 \mathrm{GHz}$. This value was obtained by interpolating the flux density measurements reported by Readhead et al. (1996). The use of the Readhead et al. (1996) values to interpolate the flux density is valid because the radio emission is dominated by extended radio lobes (see below) and not by a compact core (and thus it is less likely to exhibit strong variability). Despite the uncertainty on the flux density calibration, the peak flux density values of the narrow and broad H I lines detected in this work are very similar to those obtained with WSRT (Vermeulen et al. 2003a). In addition, our discussion of H I absorption is based on opacity considerations, which are not affected by systematic flux density calibration errors.

We transferred the self-calibration tables from the continuum data set to the line file, and subtracted the radio continuum using the task UVLIN in AIPS. We used a Brigg's robust 0 weighting for the final imaging that resulted in a $\theta_{\text {syn }}=8.3 \times 6.7$ mas $(\sim 27 \mathrm{pc}$ linear scale), $\mathrm{PA}=0.5^{\circ}, \mathrm{rms}=0.71 \mathrm{mJy}$ beam $^{-1}$ (in continuum) and $7.1 \mathrm{mJy}$ beam $^{-1}$ (per frequency channel). All velocities reported in the paper are in the rest frame of the CSO (assuming $z=0.23790$ ) unless indicated otherwise. 


\section{Results}

We detected both radio continuum and compact H I absorption in B2352+495. In Figure 1 we show the H I spectrum (top panel); the bottom panel shows the $1.14 \mathrm{GHz}$ continuum and the zero velocity moment (integrated intensity) map of the H I detection. The radio continuum has a symmetric $\mathrm{S}$-shaped distribution that is consistent with previous radio continuum images of the source (e.g., Wilkinson et al. 1994, Readhead et al. 1996, Owsianik et al. 1999). H I absorption is only detected toward the brightest continuum emission close to the center of the CSO. In Figure 2 we show the optical depth channel maps; only signal above $4 \sigma$ in the continuum and H I data were used to calculate the opacity.

The H I absorption spectrum has two features, a broad component $\left(\Delta V_{>1 \sigma}=102 \pm 8 \mathrm{~km}\right.$ $\mathrm{s}^{-1}, \mathrm{FWHM}=85 \pm 4 \mathrm{~km} \mathrm{~s}^{-1}, V_{\text {peak }}=-9.9 \pm 1.6 \mathrm{~km} \mathrm{~s}^{-1}$ ) that is almost centered at the systemic velocity of the galaxy (H I centroid Heliocentric velocity $=71307 \pm 10 \mathrm{~km} \mathrm{~s}^{-1}$; systemic velocity $\mathrm{V}_{\mathrm{Hel}}=71321 \pm 48 \mathrm{~km} \mathrm{~s}^{-1}$, optical definition), and a narrow component $\left(\mathrm{FWHM}=13 \pm 2 \mathrm{~km} \mathrm{~s}^{-1}\right)$ redshifted from systemic by $V_{\text {peak }}=129.9 \pm 0.8 \mathrm{~km} \mathrm{~s}^{-1}$. Our measurements are therefore consistent with those of Vermeulen et al. (2003a) within our velocity resolution 3

\section{Discussion}

As in the case of the H I gas associated with the nucleus of B1946+708 (Peck et al. 1999), we estimate the H I column density assuming a covering factor of 1 and a spin temperature of $8000 \mathrm{~K}$, i.e., assuming that the HI gas is directly associated with the AGN. Using the standard column density/opacity relation (e.g., Rohlfs \& Wilson 2000), we estimate a total column density of $(5.2 \pm 0.2) \times 10^{22}$ and $(7.3 \pm 1.0) \times 10^{21} \mathrm{~cm}^{-2}$ for the broad and narrow H I lines, respectively (only statistical errors from the fit were used to estimate the column density uncertainty). The values are greater than the B2352+495 H I column densities reported by Vermeulen et al. (2003a), however, as mentioned above, their values are lower limits because of the assumption of uniform coverage of the radio continuum (which is not the case, see Figure 1 ) and the assumption of a lower spin temperature $\left(T_{s p}=100 \mathrm{~K}\right)$. If the H I clouds have physical dimensions comparable to the synthesized beam (note that there appears to be structure in the opacity channels, Figure 2), then the H I density would be between $\sim 10^{2}$ and $10^{3} \mathrm{~cm}^{-3}$, corresponding to total H I masses of $\sim 2 \times 10^{5}$ and $3 \times 10^{4} \mathrm{M}_{\odot}$ for

\footnotetext{
${ }^{3}$ We note that there is a typo in the sky frequency of the narrow HI line in B2352+495 reported by Vermeulen et al. (2003a), it should read 1147.0 instead of $1147.2 \mathrm{MHz}$.
} 
the broad and narrow features, respectively.

If the H I absorption profile of the broad component is the superposition of many narrow H I features, and assuming a typical optical depth of $\tau=0.04$ for the narrow features, then we could not have detected HI absorption (at a $\geq 3 \sigma$ level) anywhere except toward the brightest continuum region at the center of the CSO (i.e., the region where H I absorption was indeed detected). This implies that, given our sensitivity limit, our data do not set significant constraints on the scale height of the H I distribution.

As discussed below, we favor the interpretation that the broad H I component is associated with rotation of a disk/torus around the supermassive black hole in B2352+495. The detection of a narrow $\mathrm{H}$ I feature redshifted with respect to the systemic velocity indicates infall. However, we cannot establish whether the H I feature is associated with circumnuclear material or with a foreground H I cloud in the host galaxy, e.g, whether it is located in the narrow-line region (NLR; e.g., B2050+364, Vermeulen et al. 2006) or associated with a high velocity cloud. We note that other studies (e.g., van Gorkom et al. 1989, Peck \& Taylor 1998, Conway 1999) have also found evidence for infalling material in galaxies with compact radio cores (see however Vermeulen et al. 2003a).

We now discuss the nature of the radio continuum having the H I absorption. In principle, the brightest $1.14 \mathrm{GHz}$ continuum source (located close to the center of the two radio lobes, Figure 1) is suggestive of the active core, i.e., the location of the supermassive black hole. However, comparing our continuum data with higher angular resolution observations reveal a different scenario. In Figure 3 (upper panel) we show our $1.14 \mathrm{GHz}$ continuum map superimposed on the $15.4 \mathrm{GHz}$ observations of Taylor et al. (1996). Both continuum data sets were self-calibrated, and thus no absolute astrometry is available. To overlay the two images, we used an average offset computed from the peak in the northern and the southern radio lobe emission; we assume that position shifts due to opacity effects are small there, since their measured separation is the same within the errors at $1.14 \mathrm{GHz}$ and $15.4 \mathrm{GHz}$. The brightest radio emission at the center of the radio lobes was not used to estimate the offset.

Taylor et al. (1996) found a compact radio source characterized by an inverted spectral index $\left(\alpha=0.14 \pm 0.3 ; S_{\nu} \propto \nu^{\alpha}\right)$, which convincingly indicates the location of the active core (see Figure 3). Assuming a flat spectral index between 1 and $8 \mathrm{GHz}$, the flux density of the core at $1 \mathrm{GHz}$ is $\sim 13 \mathrm{mJy}$. Our H I optical depth limit toward the radio core is $<0.1(3 \sigma)$. A prominent radio jet is detected to the north of the core, and a weaker counterjet to the south (Taylor et al. 1996; 2000). The northern edge of the jet is coincident with the brightest $1.14 \mathrm{GHz}$ continuum source reported in this work, implying that the $\mathrm{HI}$ absorption is not against continuum from the core, but radio emission from the jet. Among other possibilities, the detection of the radio jet and weaker counterjet could be due to relativistic beaming (see 
Taylor et al. [1996] for this and other interpretations). The HI absorption in B2352+495 is thus similar to the absorption observed in B0402+379 (Rodríguez et al. 2009) and NGC 4151 (Mundell et al. 1995, 2003) where H I is detected only toward one side of the radio jet.

It is interesting to note that the radio continuum source toward which the H I is detected (Figure 3 ) is the location where the radio jet begins to change its position angle. This would suggest that, as for example in the case of 3C 236 (Conway 1999), the H I absorption marks the location of a jet/cloud interaction responsible for disturbing (and possibly bending) the jet. However, the B2352+495 morphology is quite symmetric in both radio lobes which suggests that the deviation of the radio jets is not due to local jet/cloud interactions but governed by the large scale magnetohydrodynamical properties of the galactic nucleus. In the next section, we propose a different interpretation for the nature of the H I absorption in $\mathrm{B} 2352+495$.

\subsection{Possible H I Velocity Gradient and Disk/Torus Interpretation}

It is difficult to reliably investigate the velocity field of the broad H I line given that the absorption is only detected toward the central continuum source and, as shown in Figure 2, the velocity field is complex. Nevertheless, a velocity gradient may be present: the two channels with stronger absorption at velocities above $10 \mathrm{~km} \mathrm{~s}^{-1}$ from systemic appear to have stronger absorption toward the west, whereas most of the blueshifted absorption is stronger toward the east (velocity channels $<-10 \mathrm{~km} \mathrm{~s}^{-1}$ from systemic, see Figure 2). Figure 4 shows the position-velocity diagram of the broad H I absorption line in the eastwest direction. As also seen in the individual channel maps, there is a hint of a velocity gradient between blueshifted and redshifted material. To illustrate the possible gradient, we show in Figure 3 (top panel) the opacity channels that correspond to the rest frame velocities of 17.6 and $-31.4 \mathrm{~km} \mathrm{~s}^{-1}$ (Fig. 2).

The existence of a velocity gradient is tantalizing given that it is approximately centered at the systemic velocity of the galaxy, and the position angle of the gradient is almost perpendicular to the north-south jet, i.e., the H I gas could be tracing some kind of rotating structure around the supermassive black hole. We explore this hypothesis in the rest of this section, however, we stress that more sensitive global-VLBI observations are required to confirm the velocity gradient.

To investigate whether rotation around the supermassive back hole is physically plausible, we followed the formulation presented by Rodríguez et al. (2009; see their appendix). To model the H I rotating structure, we used the position and velocity of the peak optical 
depth of the 17.6 and $-31.4 \mathrm{~km} \mathrm{~s}^{-1}$ channels (Figure 2). The position of the radio core (Figure 3 upper panel; see also Taylor et al.1996) was assumed to be coincident with the center of mass of the system. For a black hole mass of $10^{8} \mathrm{M}_{\odot}$ (Snellen et al. 2003) and assuming circular trajectories coplanar with the supermassive black hole, we obtain that the orbit of the H I clouds from the 17.6 and $-31.4 \mathrm{~km} \mathrm{~s}^{-1}$ channels would have a radius of $\sim 20 \mathrm{pc}$ with an orbital inclination of $\sim 65^{\circ}$ with respect to the plane of the sky. Such an orbit is reasonable given the expected distribution of atomic clouds around AGNs (e.g., Peck 2005). Similar results are obtained in the case that the orbital plane of the H I clouds is not coplanar with the supermassive black hole but located above by distances of the order of the projected separation between the AGN core and the H I absorption. This estimate is clearly a zero-order approximation because non-radial inhomogeneities in the gravitational field due to stars and gas are neglected. In addition, the radius of influence of the supermassive black hole $\left(r_{B H}=G M_{B H} / \sigma_{*}^{2}\right.$, where $G$ is the gravitational constant, $M_{B H}$ is the mass of the black hole, and $\sigma_{*}$ is the stellar velocity dispersion; e.g., Neumayer et al. 2007) is $\sim 10$ pc, thus, the stellar mass enclosed by the atomic gas is likely comparable to the black hole mass.

In Figure 3 (bottom panel) we show two possibilities for the nature of the H I distribution, i.e., a thick torus (characterized by an H I scale height similar or greater than the length of the radio source) or a thin (most likely warped, e.g., Pringle 1996) disk. The obvious method to discriminate between these possibilities is to check whether there is H I toward the southern radio lobe. However, as mentioned above, our optical depth sensitivity limit does not set significant constraints on the HI distribution, i.e., clouds like those detected toward the brightest radio continuum emission could be present throughout the region.

An indirect approach to discriminate between the models is to compare our observations with lower angular resolution data. As mentioned in $§ 2$, the peak flux density measured with WSRT (Vermeulen et al. 2003a) is approximately the same peak intensity we measured with the VLBA (Figure 1) and the linewidths are also consistent. Thus, in contrast to other systems where VLBI observations resolve/filter out significant H I signal (e.g., IC 5063, Oosterloo et al. 2000), the H I in B2352+495 is compact and mostly distributed along the central continuum source, i.e., consistent with the thin disk interpretation.

The thin disk interpretation is also circumstantially supported by X-ray observations. Vink et al. (2006) reported a X-ray absorption column density of $(6.6 \pm 2.7) \times 10^{21} \mathrm{~cm}^{-2}$, which is similar to the column density of the narrow H I component, but several times smaller than the value of the broad line. If the H I absorption originates from an inclined (and warped) thin disk that does not substantially obscure the AGN (Figure 3, bottom panel; see also Fig. 5 of Orienti et al. 2006) then as observed, the column density toward the AGN derived from $\mathrm{X}$-ray observations is expected to be smaller than that of the neutral disk. 
We note that the thin disk interpretation does not necessarily imply a misalignment between the jet and the disk, i.e., the position angle of the jet changes as a function of distance from the core (Figure 3) and the disk could be warped, thus, the jet and disk could be perpendicular at (sub)parsec scales. In addition, given that the detection rate of H I absorption in CSOs is $\sim 50 \%$ (see $\S 1.1$ ), it is certainly possible that in some cases the atomic gas is distributed in a circumnuclear structure that is seen in projection only toward one radio lobe/jet.

Assuming the orbital parameters from the thin disk model, we now explore the stability of the H I disk with respect to gravitational fragmentation. We used the Toomre stability formulation as presented by Gallimore et al. (1999), i.e.,

$$
\frac{\Sigma_{H I}}{\Sigma_{c}} \simeq \frac{0.028 N_{H I} v_{r o t}}{r_{p c}}
$$

where $\Sigma_{H I}$ is the atomic hydrogen surface density, $\Sigma_{c}$ is the critical surface density above which the disk is unstable to fragmentation, $N_{H I}$ is the HI column density in units of $10^{21} \mathrm{~cm}^{-2}$, $v_{\text {rot }}$ is the rotation velocity in $\mathrm{km} \mathrm{s}^{-1}$, and $r_{p c}$ is the orbital radius in parsecs. In the case of B2352+495 we obtain $\Sigma_{H I} / \Sigma_{c} \sim 10$, thus, the disk is expected to be unstable to fragmentation. We note that reducing the assumed spin temperature by an order of magnitude would not significantly modify this conclusion, i.e., $\Sigma_{H I} / \Sigma_{c}$ would still be $\sim 1$. This simple stability test indicates that the H I cannot be distributed in a homogeneous disk. Indeed, the opacity variations from channel to channel (Figure 2) suggests that the H I gas is not homogeneously distributed. We therefore concur with the standard schematic of clumpy atomic gas around AGNs (e.g., Peck et al. 1999).

\subsection{Comparison with other High Angular Resolution Studies}

In this section we discuss the properties of $\mathrm{H}$ I absorption in B2352+495 with respect to other H I extragalactic absorbers that have been studied at very high angular resolution (i.e., interferometric observations with baselines $>100 \mathrm{~km}$; Table 1). As is clear from Table 1 and the discussion in $\$ 1.1$, high angular resolution observations of HI in CSOs reveal that the absorption does not trace a specific kind of cloud, instead, the absorbing clouds appear to be in quite different locations throughout the host galaxy, from infalling high velocity clouds and giant molecular clouds at kiloparsec scales, to torus and disks closer $(\lesssim 100 \mathrm{pc})$ to the nucleus.

Only two of the five CSOs imaged at high angular resolution exhibit HI absorption 
toward both radio lobes (Table 1; B1934-638 has not be imaged at high angular resolution, see $\S 1.1$ ). In general (four out of six, Table 1), CSOs studied at high angular resolution are characterized by a broad $\left(\Delta V \gtrsim 100 \mathrm{~km} \mathrm{~s}^{-1}\right) \mathrm{HI}$ absorption component, and tend to be multi-peaked, with narrow $\left(\Delta V<100 \mathrm{~km} \mathrm{~s}^{-1}\right)$ lines detected in five of six cases.

High angular resolution observations of HI toward non-CSO radio galaxies (Table 1) show a similar trend as the CSOs counterparts, i.e., the HI absorption appears to trace a variety of environments, from disks directly associated with the AGN to clouds at kiloparsecscale distances from the active nucleus. An apparent difference is the occurrence of H I clouds interacting with radio jets. In the case of several non-CSO with double jet/lobe radio emission, the HI appears to trace regions of interaction between atomic gas and radio jets, whereas such an interpretation has not been preferred in any of the CSOs imaged in H I at high angular resolution. This trend does not appear to be due to bias of the researchers but, in most cases, seems due to real differences in the samples. For instance, the H I absorption associated with tori/disks in CSOs is found very close $(\lesssim 100 \mathrm{pc})$ to the radio core, close to the systemic velocity, and/or has evidence of rotation. Conversely, the H I in non-CSO double jet/lobe sources with possible jet/cloud iteration is found at larger distances from the core $(\gtrsim 500 \mathrm{pc})$, shows no evidence of rotation, and/or has significantly different velocity from systemic. Although a more robust statistical sample is needed to achieve strong conclusions, the apparent absence of $\mathrm{HI}$ interaction with the radio jets supports the current view that CSOs are young radio galaxies instead of being confined by dense circumnuclear material as proposed in the frustration scenario.

Arguments for the youth scenario are mostly based on the overall radio continuum properties of the sources, power budget estimates of the jets, expansion velocities, and properties of the nuclear molecular/atomic gas. CSOs lack strong extended radio emission related to energy deposited by the jets over long periods, and VLBI studies reveal proper motions that imply short dynamic ages (of the order of $10^{3} \mathrm{yr}$, e.g., Gugliucci et al. 2005). In addition, if the advance of the radio lobes would be truly frustrated, then for them to be in ram-pressure equilibrium the density of the surrounding medium should be above $10^{2} \mathrm{~cm}^{-3}$. However, if the high density material is confined to a disk/torus then the frustration scenario is not viable. B2352+495 has evidence of being a young source based on all these arguments: it has no detected extended radio emission, the expansion velocity implies a dynamic age of $\sim 1200 \mathrm{yr}$ (Taylor et al. 2000), and, as discussed above, the high density atomic gas appears to be distributed along a disk or torus which does not interact with the radio jet. Hence, the evidence favors the youth interpretation in this case. 


\section{Summary}

We report high angular resolution $\left(\theta_{\text {syn }} \sim 8\right.$ mas) observations of $\mathrm{HI}$ in the CSO B2352+495. We detect two absorption features: a broad line almost centered at the systemic velocity of the host galaxy, and a redshifted narrow line indicating infall. Both lines are seen in absorption against the radio continuum of the central radio source of the CSO, which is the brightest continuum source in the region at the observed frequency. The radio continuum originates from the radio jet and not from the core.

In the case of the narrow HI component, our data cannot establish the location of the $\mathrm{HI}$ cloud with respect to the AGN, i.e., the radial distance of the cloud to the galaxy center is unknown. On the other hand, the broad component is likely tracing circumnuclear material close $(<100 \mathrm{pc})$ to the AGN. Even though we cannot constrain the scale height of the HI gas, the broad H I absorption could originate from a clumpy circumnuclear disk.

All available data suggest that B2352+495 is a young radio source and not an older source whose radio lobes have been confined by high density circumnuclear material. Hence, this work supports the study of CSOs as a key to understand the origin of extended radio galaxies.

We acknowledge an anonymous referee for critical comments that significantly improved the manuscript. E.D.A. was partially supported by a postdoctoral fellowship at the Univer-

sity of New Mexico. This research has made use of NASA's Astrophysics Data System, and the NASA/IPAC Extragalactic Database (NED) which is operated by the Jet Propulsion Laboratory, California Institute of Technology, under contract with NASA.

Facilities: VLBA

\section{REFERENCES}

Augusto, P. 2007, in ASP Conf. Ser. 380, At the Edge of the Universe: Latest Results from the Deepest Astronomical Surveys, ed. J. Afonso, H. C. Ferguson, B. Mobasher, \& R. Norris (San Francisco: ASP), 261

Augusto, P., Gonzalez-Serrano, J. I., Perez-Fournon, I., \& Wilkinson, P. N. 2006, MNRAS, 368,1411

Augusto, P., Wilkinson, P. N., \& Browne, I. W. A. 1998, MNRAS, 299, 1159

Beswick, R. J., Peck, A. B., Taylor, G. B., \& Giovannini, G. 2004, MNRAS, 352, 49 
Beswick, R. J., Pedlar, A., \& McDonald, A. R. 2002, MNRAS, 335, 1091

Bregman, J. N. et al. 1981, Nature, 293, 714

Carilli, C. L., \& Taylor, G. B. 2000, ApJ, 532, L95

Carilli, C. L., Wrobel, J. M., \& Ulvestad, J. S. 1998, AJ, 115, 928

Cole, G. H. J., Pedlar, A., Mundell, C. G., Gallimore, J. F., \& Holloway, A. J. 1998, MNRAS, 301,782

Conway, J. E. 1999, New Astro. Rev., 43, 509

Conway, J. E., Pearson, T. J., Readhead, A. C. S., Unwin, S. C., Xu, W., \& Mutel, R. L. 1992, ApJ, 396, 62

Cotton, W. D., Feretti, L., Giovannini, G., Venturi, T., Lara, L., Marcaide, J., \& Wehrle, A. E. 1995, ApJ, 452, 605

Curran, S. J., Whiting, M. T., Wiklind, T., Webb, J. K., Murphy, M. T., \& Purcell, C. R. 2008, MNRAS, 391, 765

De Young, D. S. 1993, ApJ, 402, 95

Fanaroff, B. L., \& Riley, J. M., 1974, MNRAS, 167, 31

Gallimore, J. F., Baum, S. A., O’Dea, C. P., Pedlar, A., \& Brinks, E. 1999, ApJ, 524, 684

Gallimore, J. F., Holloway, A. J., Pedlar, A., \& Mundell, C. G. 1998, A\&A, 333, 13

Giovannini, G., Cotton, W. D., Feretti, L., Lara, L., \& Venturi, T. 2001, ApJ, 552, 508

Giroletti, M., Giovannini, G., Taylor, G. B., Conway, J. E., Lara, L., \& Venturi, T. 2003, A\&A, 399, 889

Guainazzi, M., Siemiginowska, A., Stanghellini, C., Grandi, P., Piconcelli, E., \& AzubikeUgwoke, C. 2006, A\&A, 446, 87

Gugliucci, N. E., Taylor, G. B., Peck, A. B., \& Giroletti, M. 2005, ApJ, 622, 136

Gupta, N., Salter, C. J., Saikia, D. J., Ghosh, T., \& Jeyakumar, S. 2006, MNRAS, 373, 972

Holt, J. et al. 2006, MNRAS, 370, 1633

Labiano, A., Vermeulen, R. C., Barthel, P. D., O’Dea, C. P., Gallimore, J. F., Baum, S., \& de Vries, W. 2006, A\&A, 447, 481 
Lister, M. L., Kellermann, K. I., Vermeulen, R. C., Cohen, M. H., Zensus, J. A., \& Ros, E. 2003, ApJ, 584, 135

Neumayer, N., et al. 2007, ApJ, 671, 1329

Mirabel, I. F. 1990, ApJ, 352, L37

Momjian, E., Romney, J. D., Carilli, C. L., \& Troland, T. H. 2003a, ApJ, 597, 809

Momjian, E., Romney, J. D., Carilli, C. L., Troland, T. H., \& Taylor, G. B. 2003b, ApJ, 587,160

Morganti, R. 2004, in Proceedings of the 7th Symp. of the European VLBI Network, ed. R. Bachiller, F. Colomer, J. F. Desmurs, \& P. de Vicente (Madrid: Observatorio Astronomico Nacional), 133

Morganti, R., Oosterloo, T. A., Tadhunter, C. N., Vermeulen, R., Pihlström, Y. M., van Moorsel, G., \& Wills, K. A. 2004, A\&A, 424, 119

Morganti, R., Oosterloo, T., Tadhunter, C. N., Wills, K. A., Tzioumis, A., \& Reynolds, J. 2000, in Proc. of the 5th European VLBI Network Symp., ed. J. E. Conway, A. G. Polatidis, R. S. Booth, \& Y. M. Pihlström, ISBN 91-631- 0548-9, 111 (astro-ph/0010482)

Mundell, C. G., Ferruit, P., \& Pedlar, A. 2001, ApJ, 560, 168

Mundell, C. G., Pedlar, A., Baum, S. A., O’Dea, C. P., Gallimore, J. F., \& Brinks, E. 1995, MNRAS, 272, 355

Mundell, C. G., Wrobel, J. M., Pedlar, A., \& Gallimore, J. F. 2003, ApJ, 583, 192

O’Dea, C. P., Baum, S. A., \& Stanghellini, C. 1991, ApJ, 380, 66

O’Dea, C. P., Gallimore, J., Stanghellini, C., Baum, S. A., \& Jackson, J. M. 2005, AJ, 129, 610

Oosterloo, T. A., et al. 2000, AJ, 119, 2085

Orienti, M., Morganti, R., \& Dallacasa, D. 2006, A\&A, 457, 531

Owen, F. N., \& White, R. A. 1991, MNRAS, 249, 164

Owsianik, I., Conway, J. E., \& Polatidis, A. G. 1999, New Astro. Rev., 43, 669

Peck, A. B. 1999, PhD Thesis, New Mexico Inst. of Mining and Technology 
Peck, A. B. 2005, in ASP Conf. Ser. 340, Future Directions in High Resolution Astronomy, ed. J. Romney \& M. Reid (San Francisco: ASP), 210

Peck, A. B., \& Taylor, G. B. 1998, ApJ, 502, L23

Peck, A. B., \& Taylor, G. B. 2001, ApJ, 554, L147

Peck, A. B., Taylor, G. B., \& Conway, J. E. 1999, ApJ, 521, 103

Perlman, E. S., Carilli, C. L., Stocke, J. T., Conway, J. 1996, AJ, 111, 1839

Perlman, E. S., et al. 2002, AJ, 124, 2401

Phillips, R. B., \& Mutel, R. L. 1982, A\&A, 106, 21

Pihlström, Y. M., Conway, J. E., Booth, R. S., Diamond, P. J., \& Koribalski, B. 1999, New A Rev., 43, 647

Pihlström, Y. M., Conway, J. E., \& Vermeulen, R. C. 2003, A\&A, 404, 871

Polatidis, A. G., \& Aalto, S. 2001, in IAU Symp. 205, Galaxies and Their Constituents at the Highest Angular Resolutions, ed. R. T. Schilizzi, S. Vogel, F. Paresce, \& M. Elvis (San Francisco: ASP), 198

Polatidis, A. G., \& Conway, J. E. 2003, PASA, 20, 69

Pringle, J. E. 1996, MNRAS, 281, 357

Readhead, A. C. S., Taylor, G. B., Xu, W., Pearson, T. J., Wilkinson, P. N., \& Polatidis, A. G. 1996, ApJ, 460, 612

Rodríguez, C., Taylor, G. B., Zavala, R. T., Pihlström, Y. M., \& Peck, A. B. 2009, ApJ, 697, 37

Rohlfs, K., \& Wilson, T. L. 2000, Tools of Radio Astronomy (3rd ed., Berlin: Springer)

Rudnick, L., \& Jones, T. W. 1983, AJ, 88, 518

Sambruna, R. M., Eracleous, M., \& Mushotzky, R. F. 1999, ApJ, 526, 60

Sawada-Satoh, S., Inoue, M., Shibata, K. M., Kameno, S. \& Migenes, V. 2000, PASJ, 52, 421

Schechter, P. 1976, ApJ, 203, 297 
Snellen, I. A. G., Lehnert, M. D., Bremer, M. N., \& Schilizzi, R. T. 2003, MNRAS, 342, 889

Stanghellini, C., O’Dea, C. P. Dallacasa, D., Cassaro, P., Baum, S. A., Fanti, R., \& Fanti, C. 2005, A\&A, 443, 891

Tarchi, A., Greve, A., Peck, A. B., Neininger, N., Wills, K. A., Pedlar, A., \& Klein, U. 2004, MNRAS, 351, 339

Taylor, G. B. 1996, ApJ, 470, 394

Taylor, G. B. Marr, J. M., Pearson, T. J., \& Readhead, A. C. S. 2000, ApJ, 541, 112

Taylor, G. B., O’Dea, C. P., Peck, A. B., \& Koekemoer, A. M. 1999, apJ, 512, L27

Taylor, G. B., Peck, A. B., Ulvestad, J. S., \& O’Dea, C. P. 2004, ApJ, 612, 780

Taylor, G. B., Readhead, A. C. S., \& Pearson, T. J. 1996, ApJ, 463, 95

Tzioumis, A., et al. 2002, A\&A, 392, 841

van Breugel, W., Miley, G., \& Heckman, T. 1984, AJ, 89, 5

van Gorkom, J. H., Knapp, G. R., Ekers, R. D., Ekers, D. D., Laing, R. A., \& Polk, K. S. 1989, AJ, 97, 708

van Langevelde, H. J., Pihlström, Y. M., Conway, J. E., Jaffe, W., \& Schilizzi, R. T. 2000, A\&A, 354, L45

Vermeulen, R. C. 2002, in The Universe at Low Radio Frequencies, IAU Symp. 199, ASP Conf. Ser., ed. A. Pramesh Rao, G. Swarup, \& Gopal-Krishna (San Francisco: ASP), 91 astro-ph/0012352)

Vermeulen, R. C., Labiano, A., Barthel, P. D., Baum, S. A., de Vries, W. H., \& O’Dea, C. P. 2006, A\&A, 447, 489

Vermeulen, R. C., Ros, E., Kellermann, K. I., Cohen, M. H., Zensus, J. A., \& van Langevelde, H. J. 2003b, A\&A, 401, 113

Vermeulen, R. C., et al. 2003a, A\&A, 404, 861

Véron-Cetty, M. P., Woltjer, L., Staveley-Smith, L., \& Ekers, R. D. 2000, A\&A, 362, 426

Vink, J., Snellen, I., Mack, K.-H., \& Schilizzi, R. 2006, MNRAS, 367, 928

Waltman, E. B., et al. 1991, ApJS, 77, 379 
Wilkinson, P. N., Polatidis, A. G., Readhead, A. C. S., Xu, W., \& Pearson, T. J. 1994, ApJ, 432, L87 
Table 1. H I Observations of AGN and Starbursts at High Angular Resolution*

\begin{tabular}{|c|c|c|c|c|}
\hline Galaxy & HI Detection ${ }^{a}$ & Line Profile ${ }^{b}$ & HI Location ${ }^{c}$ & Ref. \\
\hline \multicolumn{5}{|c|}{ 1. Compact Symmetric Objects } \\
\hline 4C 31.04, B0116+319, J0119+3210 & Both lobes & $\mathrm{N}, \mathrm{B}, \mathrm{M}$ & $\begin{array}{l}\text { AGN torus }+ \\
\text { infalling HVC }\end{array}$ & 1 \\
\hline $4 \mathrm{C} 37.11, \mathrm{~B} 0402+379, \mathrm{~J} 0405+3803$ & One lobe & $\mathrm{B}, \mathrm{M}$ & AGN torus & 2 \\
\hline $\mathrm{OQ}+122$, PKS $1413+135, \mathrm{~J} 1415+1320$ & One lobe & $\mathrm{N}$ & GMC in kpc disk & 3 \\
\hline B1934-638, PKS 1934-63, J1939-6342 & $\ldots \ddagger$ & $\mathrm{N}$ & Infalling HVC? & 4 \\
\hline $\mathrm{B} 1946+708, \mathrm{~J} 1945+7055$ & Both lobes & $\mathrm{N}, \mathrm{B}, \mathrm{M}$ & AGN torus & 5,6 \\
\hline $\mathrm{B} 2352+495, \mathrm{~J} 2355+4950$ & One lobe & $\mathrm{N}, \mathrm{B}, \mathrm{M}$ & $\begin{array}{l}\text { AGN disk }+ \\
\text { infalling cloud }\end{array}$ & $\begin{array}{l}\text { This } \\
\text { work }\end{array}$ \\
\hline
\end{tabular}

2. Non-CSO Radio Sources: Double Jet/Lobe Systems as Imaged by VLBI

3C 49, B0138+136, J0141+1353

NGC 1052, B0238-084, J0241-0815

Mrk 6, IRAS 06457+7429, J0652+7425

Hydra A, 3C 218, B0915-118, J0918-1205

3C 236, B1003+351, J1006+3454

NGC 3894, B1146+596, J1148+5924

3C 268.3, B1203+6430, J1206+6413

NGC 4151, B1208+396, J1210+394

NGC 4261, B1216+061, J1219+0549

Mrk 231, IRAS $12540+5708$, J1256+5652

$4 \mathrm{C} 12.50$, IRAS $13451+1232, \mathrm{~J} 1347+1217^{\dagger}$

3C 293, B1350+3141, J1352+3126

NGC 5793, IRAS 14566-1629, J1459-1641

NGC 5929, B1524+4151, J1526+4140

PKS 1814-63, J1820-6343

IC 5063, B2048-572, J2052-5704

TXS 2226-184, IRAS 22265-1826, J2229-1810

NGC 7469, B2300+0836, J2303+0852

PKS 2322-123, J2325-1207

NGC 7674, IRAS 23254+0830, J2327+0846

$\begin{array}{ll}\text { One lobe } & \mathrm{N}, \mathrm{M} \\ \text { Both radio jets } & \mathrm{N}, \mathrm{M} \\ \text { One side of jet } & \mathrm{N} \\ \text { Both jets+core } & \mathrm{N}, \mathrm{M} \\ \text { One lobe } & \mathrm{B} \\ \text { Lobes + core } & \mathrm{N}, \mathrm{M} \\ \text { One lobe } & \mathrm{N} \\ \text { One radio jet } & \mathrm{N}, \mathrm{M} \\ \text { Counterjet+core? } & \mathrm{N}, \mathrm{M} ? \\ \text { Diffuse cont. } & \mathrm{B} \\ \text { Counterjet } & \mathrm{B}, \mathrm{M} \\ \text { Both jets+core } & \mathrm{B}, \mathrm{N}, \mathrm{M} \\ & \mathrm{N}, \mathrm{M} \\ \text { Both lobes } & \mathrm{N} \\ \text { One lobe } & \mathrm{M}, \mathrm{N} \\ \text { Both lobes } & \mathrm{B}, \mathrm{M} \\ & \mathrm{B} \\ \text { One lobe } & \mathrm{N} \\ \text { Radio jet } & \mathrm{B}, \mathrm{M} \\ & \mathrm{B}, \mathrm{N}, \mathrm{M}\end{array}$

Cloud/jet interaction 7

Nuclear and/or galactic? 8 kpc disk 9

AGN disk 10

Cloud/jet interaction $\quad 1$

AGN torus 11

Cloud/jet interaction $\quad 7$

AGN torus 12

AGN disk 13

$\sim 100$ pc disk $\quad 14$

Cloud/jet interaction $\quad 15$

Outer $(8 \mathrm{kpc})$ and $\quad 16$

$<400$ pc disk

$\sim 1$ kpc torus? $\quad 17$

AGN ring, or bar? $\quad 18$

AGN disk + 19

Cloud/jet interaction?

Cloud/jet interaction 20

Nuclear region, $\quad 21$

cloud/jet interaction?

Nuclear or galactic? 22

AGN disk + infall? $\quad 23$

AGN disk/torus 24
NGC 315, B0055+300, J0057+3021

NGC 3079, IRAS 09585+5555, J1001+5540

Cen A, NGC 5128, B1322-428, J1325-4301

PKS 1549-79, B1549-790, J1557-7913

Cygnus A, IRAS 19577+4035, J1959+4044

DA 529, B2050+364, J2052+3635

$\begin{array}{ll}\text { One jet }+ \text { core } & \mathrm{N} \\ \text { One jet }+ \text { core } & \mathrm{N}, \mathrm{M} \\ \text { One jet } & \mathrm{N}, \mathrm{M} \\ \text { One jet }+ \text { core } & \mathrm{N} \\ \text { Counterjet }+ \text { core? } & \mathrm{B} \\ \text { One jet }+ \text { core } & \mathrm{N}, \mathrm{M}\end{array}$

Infalling cloud

AGN torus

kpc disk or rings

Nuclear region?

Torus or $2 \mathrm{kpc}$ ring

NLR/BLG $(<1 \mathrm{kpc})$ 
Table 1-Continued

\begin{tabular}{|c|c|c|c|c|}
\hline Galaxy & HI Detection ${ }^{a}$ & Line Profile ${ }^{b}$ & HI Location ${ }^{c}$ & Ref. \\
\hline \multicolumn{5}{|c|}{ 4. Non-CSO Radio Sources: Starbursts } \\
\hline NGC $2146,4 \mathrm{C}+78.06, \mathrm{~B} 06106+7822, \mathrm{~J} 0618+7821$ & Starburst cont. & $\mathrm{B}, \mathrm{N}, \mathrm{M}$ & Disk or ring & 29 \\
\hline IC $694, \operatorname{Arp} 299$, Mrk 171, B1125+588, J1128+5834 & Starburst cont. & $\cdots$ & $250 \mathrm{pc}$ disk & 30 \\
\hline Mrk 273, B1342+5608, J1344+5553 & Starburst cont. & $\mathrm{B}, \mathrm{M}$ & Starburst cores & 31 \\
\hline Arp 220, B1532+2339, J1534+2330 & Starburst cont. & $\mathrm{N}, \mathrm{B}, \mathrm{M}$ & Disks in starburst cores & 32 \\
\hline PGC 060189, IRAS 17208-0014, J1723-0016 & Starburst cont. & $\mathrm{B}, \mathrm{N}, \mathrm{M}$ & Nuclear $\sim 1 \mathrm{kpc}$ & 33 \\
\hline
\end{tabular}

Note. _ * Interferometric observations with baselines greater than $100 \mathrm{~km}$. The sources are divided in four groups, CSOs, non-CSO radio sources with detection of two radio jets or lobes in opposite sides of a central core, non-CSO radio sources dominated by core-jet emission (although a weak counterjet may be detected, e.g., Cygnus A, Conway 1999), and starburst galaxies. (a) Indicates whether HI absorption was detected towards all radio continuum emission (both lobes and core, or starburst), or only towards one radio lobe. ${ }^{(b)}$ Detection of broad lines $\left(\Delta V>100 \mathrm{~km} \mathrm{~s}^{-1}\right)$ is indicated with 'B', narrow lines $\left(\Delta V<100 \mathrm{~km} \mathrm{~s}^{-1}\right)$ with ' $\mathrm{N}$ ', multiple lines in the spectrum (overlapped or otherwise) with 'M'. (c) Most likely (or at least preferred) location of the HI material with respect to the AGN as discussed in the reference paper, e.g., associated with a disk of torus within $\sim 100 \mathrm{pc}$ from the AGN, with giant molecular clouds (GMC) or high velocity clouds (HVC) at galactic (kiloparsec) scales. ${ }^{\ddagger}$ ) The HI distribution in this source has not been imaged with VLBI, however, the absorption is against the compact radio continuum given detection of $\mathrm{HI}$ with three LBA baselines (Véron-Cetty et al. 2000; see also $\S 1.1$ ). ( $\dagger$ ) The classification of $4 \mathrm{C}+12.50$ is controversial, see $\S 1.1$. - References: 1. Conway (1999), 2. Rodríguez et al. (2009), 3. Perlman et al. (2002), 4. Véron-Cetty et al. (2000), 5. Peck et al. (1999), 6. Peck \& Taylor (2001), 7. Labiano et al. (2006), 8. Vermeulen et al. (2003b), 9. Gallimore et al. (1998), 10. Taylor (1996), 11. Peck \& Taylor (1998), 12. Mundell et al. (2003), 13. van Langevelde et al. (2000), 14. Carilli et al. (1998), 15. Morganti et al. (2004), 16. Beswick et al. (2004), 17. Pihlström et al. (1999), 18. Cole et al. (1998), 19. Morganti et al. (2000), 20. Oosterloo et al. (2000), 21. Taylor et al. (2004), 22. Beswick et al. (2002), 23. Taylor et al. (1999), 24. Momjian et al. (2003a), 25. Peck (1999; see also Morganti 2004), 26. Sawada-Satoh, et al. (2000), 27. Holt et al. (2006), 28. Vermeulen et al. (2006), 29. Tarchi et al. (2004), 30. Polatidis \& Aalto (2001), 31. Carilli \& Taylor (2000), 32. Mundell et al. (2001), 33. Momjian et al. (2003b). 

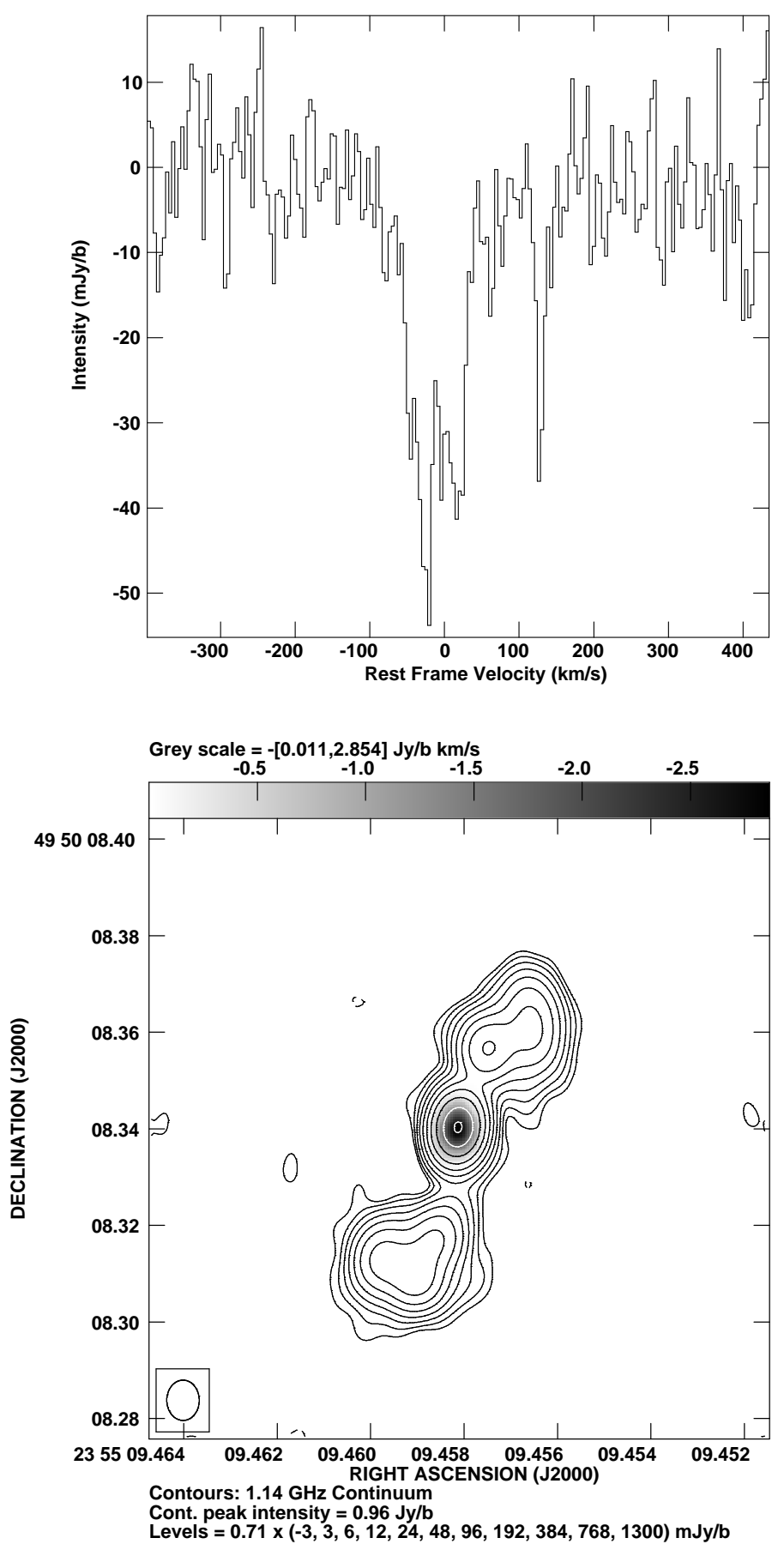

Fig. 1. - Top: H I absorption detected toward the compact symmetric object B2352+495 after continuum subtraction. The systemic velocity of the system $\left(\mathrm{V}_{\mathrm{Hel}}=71321 \pm 48 \mathrm{~km} \mathrm{~s}^{-1}\right.$, $V=0.0 \mathrm{~km} \mathrm{~s}^{-1}$ at the rest frame of the galaxy) is almost at the centroid of the broad absorption feature. Bottom: Zero velocity moment (integrated intensity) of the H I absorption (grey scale) and $1.14 \mathrm{GHz}$ continuum (contours). The data were self-calibrated and thus have no absolute astrometry; the radio continuum peak was assigned to the phase tracking center $\left(\mathrm{RA}=22^{\mathrm{h}} 55^{\mathrm{m}} 09.4581^{\mathrm{s}}\right.$, Decl. $=+49^{\circ} 50^{\prime} 08.340^{\prime \prime}$, J2000). There is no registration difference between the continuum and H I absorption. 


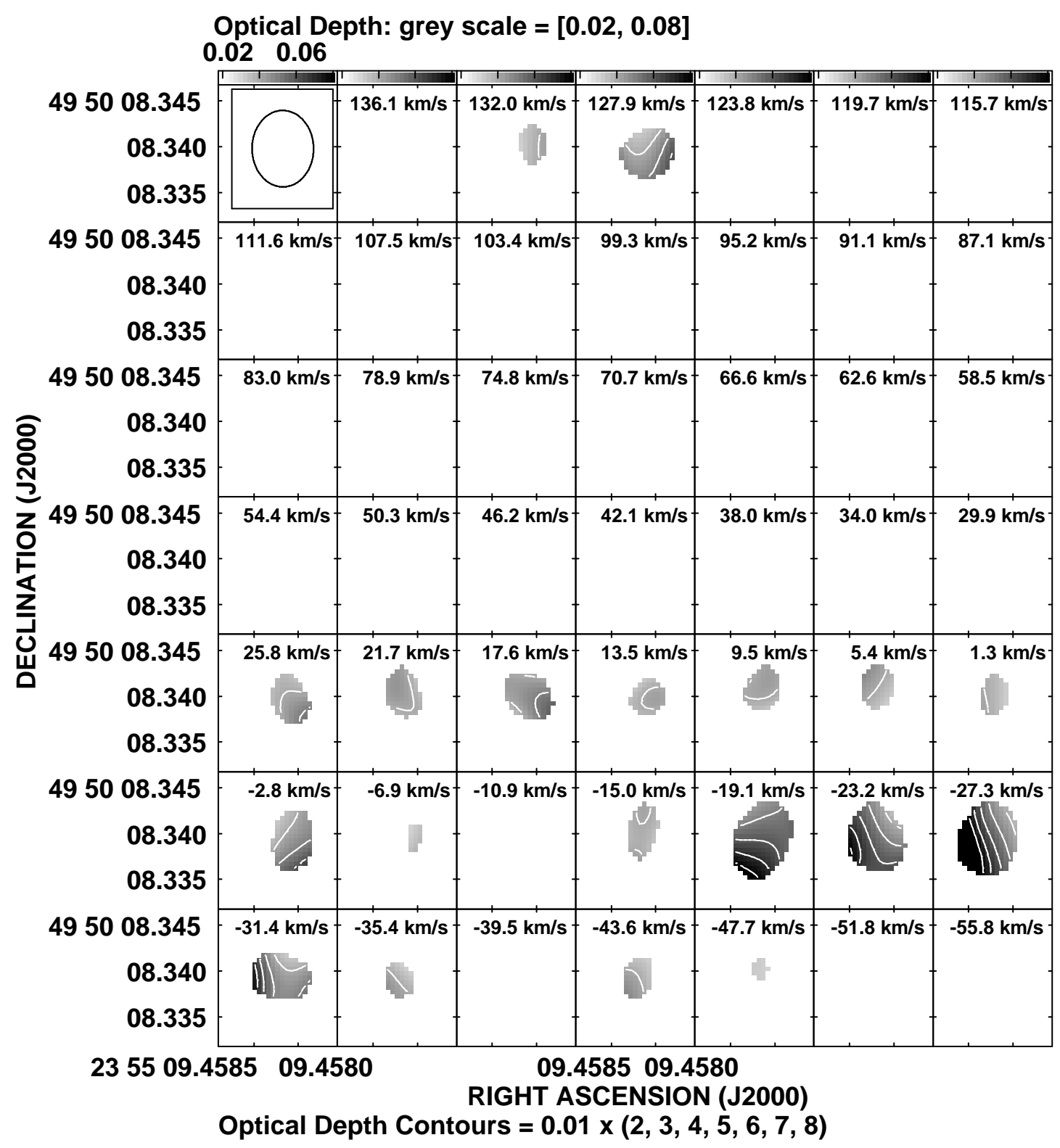

Fig. 2.- Optical depth channel maps (rest frame velocity is given). The area in each channel map corresponds to the central region of B2352+495 where H I was detected (compare the synthesized beam size shown in the top-left panel with the synthesized beam shown in Figure 1, bottom panel). Only H I absorption with absolute magnitude greater than $4 \sigma$ was used to calculate the optical depth. 

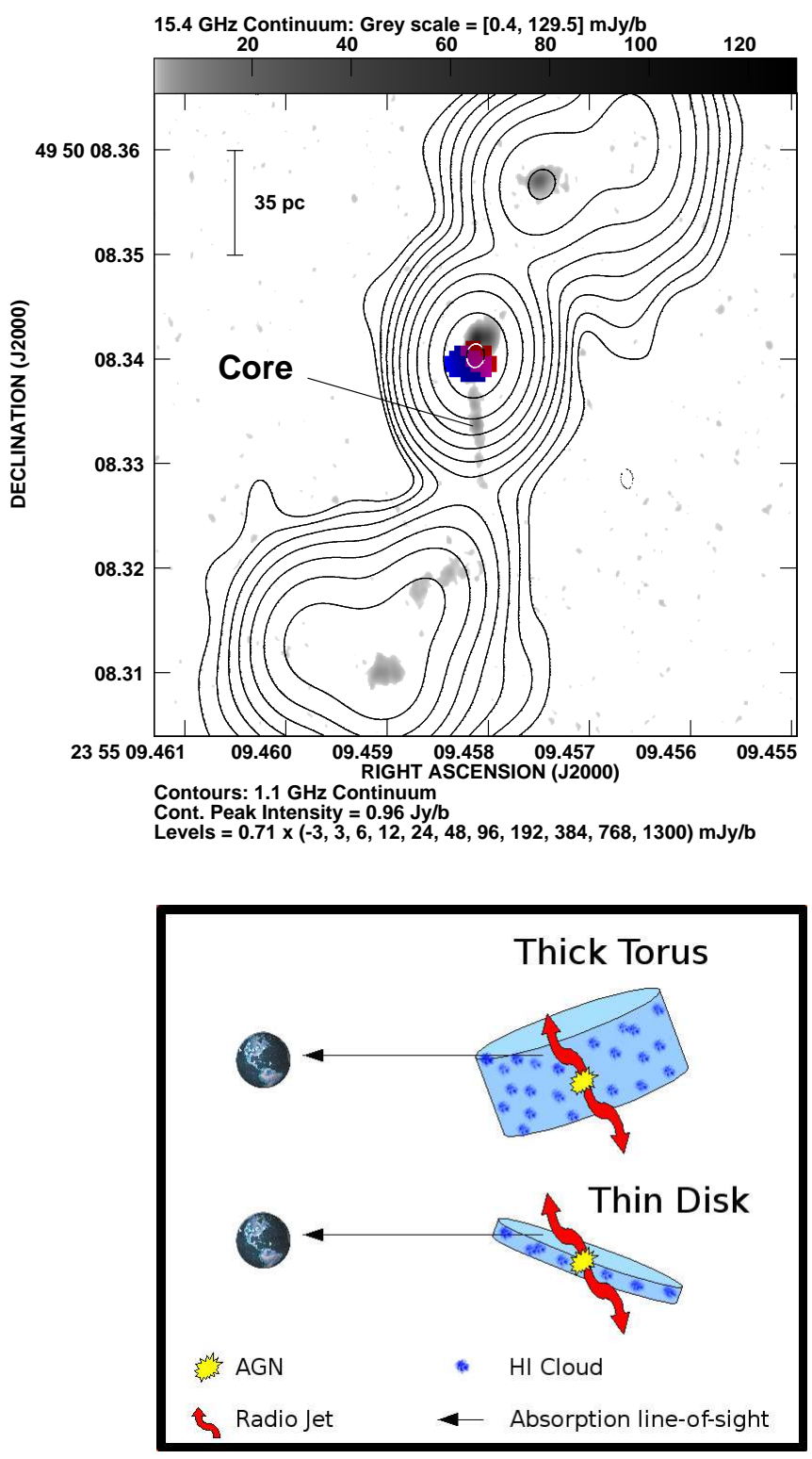

Fig. 3.- Top: Zoomed in image of the radio continuum in B2352+495 at $15.4 \mathrm{GHz}$ (grey scale; Taylor et al. 1996) and $1.14 \mathrm{GHz}$ (contours; this work). Taylor et al. (1996) found that the center of activity (radio core) is located $\sim 20 \mathrm{pc}$ south of the brightest $15.4 \mathrm{GHz}$ radio continuum source. In red and blue we show the H I optical depth channels that correspond to the rest frame velocities of 17.6 and $-31.4 \mathrm{~km} \mathrm{~s}^{-1}$ (see Figure 2; the opacity range of the red and blue channels is as in Figure 2, i.e., 0.02 to 0.08). We found a tentative H I velocity gradient that is almost perpendicular to the jet direction. Bottom: Two possible interpretations of the H I absorption in B2352+495: a thick torus or a thin (possibly warped) disk. The red arrows represent the flow direction in the jets. The line of sight to Earth (shown with the symbol taken from the VLBA/NRAO logo) is in the plane of the picture. Radio continuum from parts of the jets directed more towards Earth will appear brightened due to Doppler boosting. 


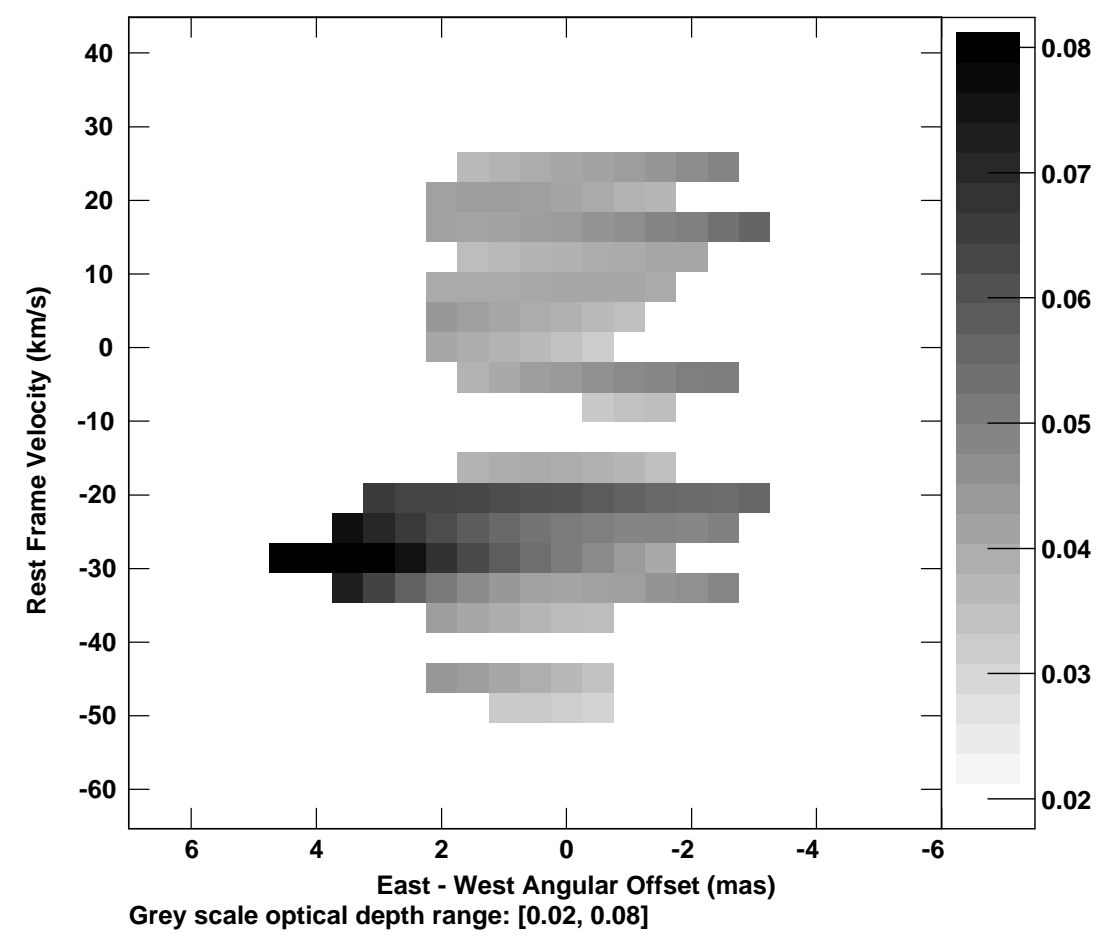

Fig. 4.- Position-velocity (P-V) diagram of the broad H I absorption component. The P-V diagram is along a east-west position angle passing through the center of the $\mathrm{HI}$ absorption (Figure 1). Absorption along the declination axis was averaged to increase signal-to-noise. 